\title{
POTENTIAL AND LIMITATIONS OF TERRESTRIAL LASER SCANNING FOR DISCONTINUITY ROUGHNESS ESTIMATION
}

\author{
M. Bitenc ${ }^{\mathrm{a}, *}$, D. S. Kieffer ${ }^{\mathrm{a}}$, K. Khoshelham ${ }^{\mathrm{b}}$ \\ ${ }^{a}$ Institute of Applied Geosciences, Graz University of Technology, Austria - (bitenc, kieffer)@tugraz.at \\ ${ }^{\mathrm{b}}$ Department of Infrastructure Engineering, The University of Melbourne, Victoria 3010, Australia - k.khoshelham@unimelb.edu.au
}

\author{
Commission III, WG III/2
} KEY WORDS: Terrestrial Laser Scanning, Range noise, Effective resolution, Discontinuity roughness, Stationary Wavelet
Transform

\begin{abstract}
:
Terrestrial Laser Scanning (TLS) greatly facilitates the acquisition of detailed and accurate 3D measurements of remote rock outcrops, at an operational range from several meters to a few kilometres. Reliable, quantitative measures of rock discontinuity roughness are necessary to characterize and evaluate the mechanical and hydraulic behavior of the rock mass. The aim of this research is to investigate the TLS potential and limitations for a reliable estimation of small scale roughness. TLS data noise and resolution define the level of extractable morphological detail, and therefore need to be known and associated with roughness value. The stationary variant of Discrete Wavelet Transform (SWT) was applied to estimate TLS noise level and perform wavelet denoising in direction of range measurements. Denoised TLS data were compared to reference surfaces of decreasing resolution (reference grids) in order to define the size of extractable surface detail. Noise and resolution effect on rock surface roughness, wavelet denoising success and extractable roughness scale were investigated with comparative analyses of TLS and reference surfaces. The developed methodology enabled reasonable TLS noise estimation, improved capabilities of TLS for modelling fine features of an irregular rock surface, and indicated the surface scale that can be reliably extracted from the TLS data.
\end{abstract}

\section{INTRODUCTION}

Advantages of Terrestrial Laser Scanning (TLS) are acknowledged in many fields of geological engineering, since it permits an in-situ acquisition of a large and remote surface in a short period of time, and represents the 3D surface structure with a relatively dense and precise point cloud (e.g. Buckley et al., 2008). For in-situ roughness acquisition, traditional contact measurement techniques such as linear mechanical profiling using either a straight edge or a profile gauge, and compass and disc-clinometer method have been successfully replaced by TLS (e.g. Fardin et al., 2004; Khoshelham et al., 2011). Joint surface roughness refers to local departures of the actual surface from planarity or any higher order reference surface. Roughness can have a prevailing influence on the shear strength. However, the parameterization of roughness, to fully capture the influence of roughness on shear strength, remains a challenge; it needs to consider that roughness is direction and scale dependent (Rengers, 1970). Therefore, measurements need to performed in the anticipated shear direction and at the engineering scale of interest. Larger scale roughness features are referred to as waviness and represent surface irregularities with a wavelength greater than about $10 \mathrm{~cm}$ (Priest, 1993). Smaller scale features are referred to as unevenness and include finer features that are superimposed on the waviness. While decent results have been obtained in quantifying waviness (Fardin et al., 2004), finer details of unevenness have been hindered by TLS data precision and resolution.

The data precision mainly depends on the inherent random range error (noise), which results in an overestimation of surface roughness (e.g. Kulatilake et al., 2006; Poropat, 2009; Khoshelham et al., 2011). We refer to this as the noise effect. TLS data resolution defined as the ability to distinguish surface details on adjacent line of sights (hereafter referred to as effective resolution) is a function of sampling interval and laser beam footprint size (Lichti and Jamtsho, 2006). Decreasing resolution, i.e. increasing the sampling interval, results in effective smoothing of discontinuity surface and therefore causes roughness underestimation; we refer to this as the resolution effect. Ignoring the variation of measurement resolution leads to misleading roughness estimation (Tatone and Grasselli, 2012). TLS noise and effective sampling interval increase with the scanning range and incidence angle. In general, it is neither possible to extract surface features that have an amplitude smaller than the TLS noise, nor features that are smaller than twice the data resolution (Nyquist theorem).

The aim of this research is to analyse effects of TLS noise and resolution on rock discontinuity roughness as a function of range. The objectives are to:

- Estimate and eliminate TLS noise using an advanced signal processing technique, the wavelet transform, to enable precise and detailed roughness estimation. The challenge of wavelet denoising is to eliminate noise, but preserve surface details.

- Investigate TLS effective resolution in relation to irregular rock surface and define the size of the smallest surface detail extractable from denoised surfaces.

\section{TLS NOISE ESTIMATION AND ELIMINATION}

TLS noise, defined among others in (Soudarissanane et al., 2011), can be estimated empirically by (i) scanning an object with known geometric properties, as for example a uniform plane (Vezočnik and Bitenc, 2011), cylinder or sphere, (ii) applying modeling strategies for TLS 3D point clouds (Gordon and Lichti, 2007), or (iii) using reference measurements or a model; or theoretically by using the random error propagation law (Hartzell et al., 2015; Mikhail, 1976). The range error has 
been modelled as a function of local scanning geometry (range and incidence angle) and TLS specifications (beam width) (Soudarissanane et al., 2011) or as a function of backscattered signal strength (Wujanz et al., 2017).

Noise has typically been removed by surface interpolation techniques such as averaging the TLS range measurements (Schulz et al., 2008), orthogonal least squares (Fardin et al., 2004; Pollyea and Fairley, 2011), the robust interpolation method RANSAC (Grasselli et al., 2002) or Fast Radial Basis Function (Rahman et al., 2006; Tesfamariam, 2007). Interpolation methods improve the positional accuracy of simple surfaces that can be approximated with planes but smooth out topographic details of irregular rock surfaces. Reducing the spatial complexity of a 3D randomly scattered point cloud by gridding to a regular 2.5D mesh, a wide range of image processing algorithms can be applied (Buades et al., 2005; Salmon, 2010; Smigiel et al., 2011; Zhang et al., 2014). To improve surface roughness estimation, previous researchers investigated a transforming domain filtering method, namely the DWT (Bitenc et al., 2015a, 2015b, 2019; Khoshelham et al., 2011), and a spatial (pixel) domain filtering method, namely the Non-Local Mean (NLM) (Bitenc et al., 2016; Smigiel et al., 2011). Prior research has focused on denoising in a direction perpendicular to the best fit plane. However, denoising in the range direction (hereafter referred to as range denoising) is considered preferable (Schulz et al., 2008; Smigiel et al., 2011), since noise mainly relates to the range. In this research, the TLS noise is estimated and eliminated in the range measurement direction by applying DWT. Details on the wavelet denoising can be found in numerous literature, among others in (Donoho, 1995). Based on our previous research (Bitenc et al., 2019), the optimal choices for DWT method and threshold value are as follows.

An undecimated version of DWT, referred to as the Stationary Wavelet Transform, or SWT (Buades et al., 2004; Coifman and Donoho, 1995) has been chosen. It is shift invariant, provides more precise information regarding frequency localization, enables direct correlation of the space scale to the original data, reduces the overshoot and undershoot of the signal near discontinuities (Buades et al., 2004) and shows superior performance for image denoising (Gyaourova et al., 2002; Starck et al., 2004). For denoising rock surfaces in range direction, 2D SWT is used, where the input signal is a $2.5 \mathrm{D}$ grid of range values (a range image).

The optimal threshold value $(T)$ is a key to successful wavelet denoising, and is in general calculated as:

$$
T=\sigma \times T_{0}
$$

where $\sigma$ is the standard deviation of the noise and $T_{0}$ is the threshold chosen according to one of the threshold selection methods for a signal model including white noise $(\sigma=1)$. The global penalised (Birgé and Massart, 1997) threshold selection methods is applied, setting its adjustable (sparsity) parameter to 6.5 (penalized high). Higher sparsity parameter returns higher threshold, thus more coefficients are eliminated resulting in a sparser (smoother) signal representation. The threshold is applied in hard mode, following previous findings that hard thresholding is more suitable for rock surface roughness estimation than soft thresholding (Bitenc et al., 2015a; Khoshelham et al., 2011)

Considering the statistical property of the DWT that transforms white noise of the input data into white noise of the output data, the actual noise can be estimated from the variance of detail coefficients on level $j, c D_{j}$, corresponding to the noise. The first level detail coefficients, $c D_{1}$, having the finest scale and being least decimated, are often considered as noise coefficients. Therefore, a robust standard deviation of $c D_{1}$, referred to as the Median Absolute Deviation (MAD), is used to estimate the noise $\sigma_{e}$ as (Donoho and Johnstone, 1995):

$$
\sigma_{e}=\operatorname{median}\left(\left|c D_{1}\right|\right) / 0.6745
$$

The experiment with simulated TLS data, when a random Gaussian noise has been added to the reference data, has shown that the $\sigma_{e}$ equals the known noise level (Bitenc et al., 2015a). The investigation of $\sigma_{e}$ dependency on the recorded intensity (Bitenc et al., 2019), has confirmed the one term power model as developed in (Wujanz et al., 2017); $\sigma_{e}$ is inversely proportional to the mean intensity and tends to increase with the range. The advantage of $\sigma_{e}$ is, it can be estimated from raw TLS data.

\section{TLS EFFECTIVE RESOLUTION}

TLS effective resolution is defined as the level of detail or the size of an object that can be distinguished in the angular direction (perpendicular to the line of sight). A very high point density can be achieved by repeating the scans or by decreasing the angular sampling interval. Such measurements result in correlated sampling (overlapping footprints), which yields a high Nyquist frequency, but do not improve the effective resolution. Besides the angular sampling interval $\Delta$, the footprint size $\delta$ has a large impact on the effective resolution (Lichti and Gordon, 2004). $\Delta$ is usually given in scanner specifications, whereas the complete information on $\delta$ is usually missing. Neglecting internal processing of laser beam, since it is often not known, the calculation of $\delta$ for long ranges $R$, when linear dispersion of the laser beam can be assumed, and for perpendicular scanning direction $\left(\alpha=0^{\circ}\right)$ is simplified to (Lichti and Jamtsho, 2006):

$$
\delta[\mathrm{mm}]=R[\mathrm{~m}] \times \beta[\mathrm{mrad}]+\beta_{0}[\mathrm{~mm}],
$$

where $\beta$ is the beam divergence given in mili radians and $\beta_{0}$ is the opening aperture.

An Average Modulation Transfer Function (AMTF) was used to model the positional uncertainty of TLS angle measurements due to the $\Delta, \delta$ and angle quantization and a new measure for effective resolution called Effective Instantaneous Field Of View (EIFOV) was derived (Lichti and Jamtsho, 2006). Resulting EIFOV was much coarser than the specified smallest $\Delta$, pointing out the necessity to consider the $\delta$. Contribution of the angle quantization was minor and could be neglected. Analysing TLS data acquired on a test target comprising small blocks separated for a certain gap, the EIFOV value was found to be too pessimistic (Pesci et al., 2011). In their conclusions, a gap larger than one third of $\delta$, calculated after Eq. (3), could be extracted. However, when observing details of complex surfaces (e.g. building facade), the sampling interval should have been smaller than in case of gaps between blocks.

These findings suggest that the effective resolution is an application dependent measure and should be defined in accordance with the surface detail of interest. Observing details that can be approximated with simple geometric shapes (e.g. lines or planes), this additional geometric information increases the effective resolution compared to a case of extracting details on an irregular and complex surface. An empirical method is 
developed here, were a TLS surface acquired at certain range and assumed to be noise free is compared to reference surfaces of changing resolution. The effective resolution or the size of the smallest extractable rock surface detail $\lambda_{\min }$ equals the reference resolution, for which the surfaces are the most similar.

\section{EXPERIMENTS AND RESULTS}

The experiments described below were conducted to estimate noise from the TLS data and eliminate it, and to define the size of the smallest extractable surface detail, assuming that the noise was successfully removed.

\subsection{Data acquisition}

The terrestrial laser scanner Riegl VZ400 and the optical 3D coordinate measuring sensor GOM ATOS I were employed for data collection (Fig. 1). Key technical specifications of these sensors are summarized in Table 1.

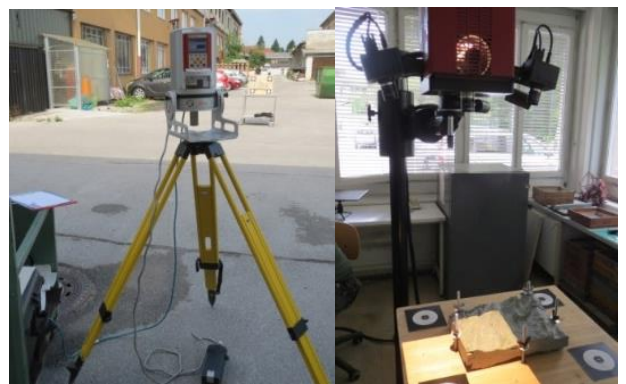

Fig. 1. The Riegl VZ400 (left) and GOM ATOS I (right) measurement set-ups

\begin{tabular}{ccc}
\hline & Riegl VZ400 & GOM ATOS I \\
\hline $\begin{array}{c}\text { Measurement } \\
\text { range }\end{array}$ & 1.5 to $500 \mathrm{~m}^{1}$ & 0.65 to $1 \mathrm{~m}$ \\
$\begin{array}{c}\text { Precision / } \\
\text { accuracy }\end{array}$ & $3 \mathrm{~mm} / 5 \mathrm{~mm}$ & $\begin{array}{c}0.007 \text { to } 0.07 \\
\mathrm{~mm} / \text { Unknown }\end{array}$ \\
\hline $\begin{array}{c}\text { Spot or pixel size } \\
\text { Point spacing }\end{array}$ & $\begin{array}{c}6.5 \mathrm{~mm} \text { beam aperture } \\
+0.3 \mathrm{mrad} \text { divergence } \\
\text { min. } 0.0024^{\circ}\end{array}$ & 0.04 to $1 \mathrm{~mm}$ \\
\hline $\begin{array}{c}\text { Measurement rate } \\
\text { [points } / \mathrm{s} \text { ] }\end{array}$ & $\begin{array}{c}42 \mathrm{k}(\text { long range) or } \\
122 \mathrm{k}(\text { high speed) }\end{array}$ & 0.04 to $1 \mathrm{~mm}$ \\
\hline $\begin{array}{c}\text { Measuring FOV or } \\
\text { area }\end{array}$ & $360^{\circ} \times 100^{\circ}$ & $30 \times 40 \mathrm{~mm}$ to \\
\hline at $80 \%$ reflectivity \\
Table 1. Specifications of Riegl VZ400 and GOM ATOS I.
\end{tabular}

The four rock samples comprising the experimental data set are shown in Fig. 2. For each sample the TLS scans were acquired at $10 \mathrm{~m}$ intervals in the range of $10 \mathrm{~m}$ to $60 \mathrm{~m}$, denoted by $R_{k}$ $(k=1 . .6)$. The scanning resolution on the rock surface ranged from approximately 0.2 to $2 \mathrm{~mm}$. For a filtered 3D point cloud, Cartesian $(X, Y, Z)$ and polar $(\Phi, \Theta, R)$ coordinates along with raw intensity values were exported in the scanner coordinate system.

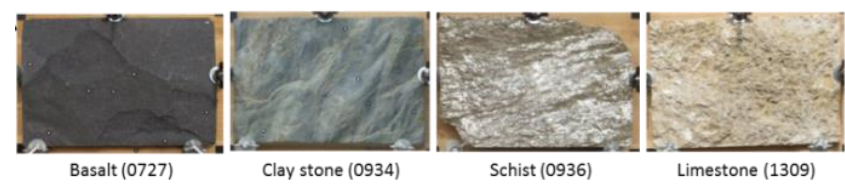

Fig. 2. Rock samples, $20 \times 30 \mathrm{~cm}$ in plan dimensions and mounted to a wooden board

ATOS data were acquired at a range of approximately $0.7 \mathrm{~m}$ in an indoor laboratory environment. To eliminate the influence of data resolution on roughness comparisons, the high ATOS point density was reduced to the TLS point density of approximately 1 point $/ \mathrm{mm}^{2}$ using the subsample tool in CloudCompare (CloudCompare, 2018).

Four targets were established on the sample mounting board in order to co-register the TLS and ATOS data. High precision TLS target centers were measured in the point cloud by applying an algorithm based on image matching (Kregar et al., 2013) and ATOS target centres were identified automatically with built-in software.

\subsection{Data processing}

For TLS noise estimation and 2D wavelet denoising in range direction, randomly scattered TLS points $(\Phi, \Theta, R)$ were interpolated into a regular grid (range image) with an angular spacing equal to $1 \mathrm{~mm}$ at the corresponding scanning range. The range value was interpolated using the Nearest Neighbour (NN) method. Range image was decomposed with SWT, applying the most general and widely used Daubechies wavelet db3. The number of decomposition levels was determined according to the range image size and was set to 3 . First level detail coefficients $c D_{1}$ were used to estimate the noise $\sigma_{e}$ after Eq. (2). Further, the SWT detail coefficients were thresholded in the hard mode with the global penalized high threshold. Range denoised images were transformed to Cartesian coordinates. The TLS data, noisy and range denoised, and the ATOS data were then co-registered in a common coordinate system, which was defined by the targets on the wooden board, and point clouds within an identical rectangular area were used for roughness comparisons.

The empirical roughness parameter developed by Grasselli (2001), hereafter referred to as the Grasselli parameter, has been adopted in this research, since it quantifies the direction dependence of roughness, is applicable to rock surfaces presented in $2.5 \mathrm{D}$, and is least sensitive to data noise (Bitenc et al., 2015c). It was calculated for 72 analysis directions $\left(\gamma_{i}\right.$ $i=1 \ldots 72$ ). The accuracy of roughness estimates was judged by comparing the Grasselli parameters, $G_{k}$, of noisy and denoised TLS surfaces acquired at $R_{k}$ to Grasselli parameter, $G_{A T O S}$, for the ATOS data. A mean relative difference across all analysis directions was calculated as:

$$
\operatorname{error}_{k}[\%]=\frac{1}{72} \sum_{i=1}^{72}\left(\frac{G_{k}-G_{A T O S}}{G_{A T O S}}\right)_{\gamma i} \times 100
$$

For effective resolution analysis, ATOS data were interpolated with NN method into 17 reference grids with grid sizes $\lambda_{j}$ $(j=1 \ldots 17)$ increasing from $0.5 \mathrm{~mm}$ to 47,49 and $53 \mathrm{~mm}$, for samples 0727, 0934 and 0936, and 1309, respectively. For each sample, $\lambda_{j}$ were defined such that all grids covered the same area. Reference grids simulate the TLS data of changing effective resolution.

To estimate the smallest extractable surface detail size $\lambda_{\text {min }}$, the denoised TLS surfaces smoothed out due to the unknown effective resolution were compared to each reference grid. The $\lambda_{j}$, for which the surfaces were the most similar, represents $\lambda_{\min }$. The investigated similarity measures were the:

a) height differences $\Delta Z_{k, j}$ between denoised TLS point clouds and reference grids, and

b) mean relative differences error $_{k, j}$ of Grasselli parameters calculated for reference grids, $G_{j}$, and $G_{k}$, which is expressed as: 


$$
\operatorname{error}_{k, j}[\%]=\frac{1}{72} \sum_{i=1}^{72}\left(\frac{G_{k}-G_{j}}{G_{j}}\right)_{\gamma i} \times 100
$$

We assumed that $\lambda_{j}$ when the error $_{k, j}$ equals zero or the standard deviation of $\Delta Z_{k, j}, \operatorname{std}\left(\Delta Z_{k, j}\right)$, reaches its minimum, represents the $\lambda_{\min }$ at the $R_{k}$. A linear functional relationship between $\lambda_{\min }$ values, calculated for both similarity measures, and EIFOV, calculated for $\Delta=1 \mathrm{~mm}$ and $\delta$ obtained after Eq. (2), was analysed.

\subsection{Results and discussion}

The main results concerning TLS noise and resolution effect on Grasselli parameter, noise estimation and elimination, and effective resolution analysis are summarized below.

4.3.1 TLS noise and resolution effect. The Grasselli parameter was computed for reference ATOS data $\left(G_{\text {ATOS }}\right)$ in order to obtain an insight into the sample roughness characteristics and facilitate roughness comparisons. Table 2 summarizes the approximate size of the analysed area, and median and robust standard deviation (robust STD) values of $G_{\text {ATOS }}$ for all analysis directions. Sample 1309 is the roughest, with the median value almost three times higher than for the smoothest sample 0727. Sample 1309 also has the least variability with analysis direction as indicated by the low robust STD. The robust STD for sample 0936 shows the highest roughness anisotropy.

\begin{tabular}{ccc}
\hline Sample & $\begin{array}{c}\text { Analyzed area } \\
{[\mathrm{mm}]}\end{array}$ & $\begin{array}{c}\text { Median } \pm \text { robust STD } G_{\text {ATOS }} \\
{\left[{ }^{\circ}\right]}\end{array}$ \\
\hline 0727 & $203 \times 143$ & $7.6 \pm 2.5$ \\
0934 & $244 \times 148$ & $11.8 \pm 3.2$ \\
0936 & $217 \times 147$ & $14.3 \pm 5.7$ \\
1309 & $214 \times 161$ & $23.4 \pm 0.8$ \\
\hline
\end{tabular}

Table 2. The size of analysed area, and the median and robust STD of reference ATOS Grasselli parameters $\left(G_{\text {ATOS }}\right)$ calculated for 72 analysis directions

To study the TLS noise and resolution effect on Grasselli parameter as a function of $R$, the error $_{k}$ was calculated for TLS noisy surfaces and is shown in Fig. 3.

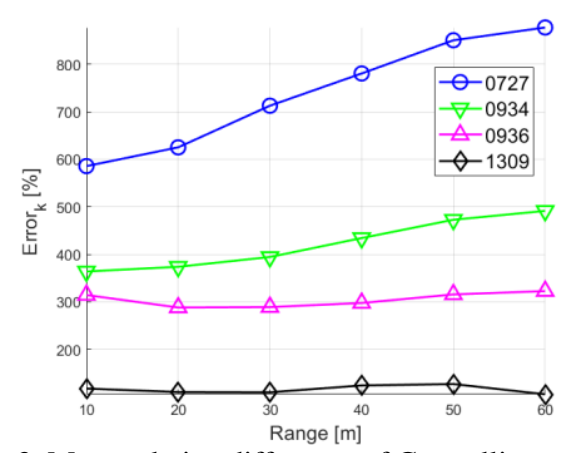

Fig. 3. Mean relative difference of Grasselli parameters $\left(\right.$ error $\left._{k}\right)$ for noisy TLS surfaces of the four samples versus range

The Grasselli parameter is systematically overestimated for all four samples. Effects depend on surface reflectivity and roughness, and $R$. The darkest and smoothest sample 0727 is most severely influenced by the noise, while the bright and roughest sample 1309 is least influenced. For sample 0727, the error $_{k}$ increases with the $R$, which is not the case for sample
1309 ; its noise effect is balanced with the smoothing effect that results from decreasing effective resolution with increasing $R$. The error $_{k}$ for samples 0727 and 1309 range from approximately 590 to $850 \%$ and 105 to $130 \%$, respectively.

4.3.2 Noise estimation: The dependency of the $\sigma_{e}$, estimated using Eq. (2), on $R$ is shown in Fig. 4. For all samples, $\sigma_{e}$ tends to increase with the $R$. Exception is the low $\sigma_{e}$ for sample 1309 scanned at $60 \mathrm{~m}$. Possible reason for this anomaly includes the low original point density of $2 \mathrm{~mm}$ compared to $1 \mathrm{~mm}$ range image pixel size. The $\sigma_{e}$ is higher for darker rock surfaces of samples 0727 and 0934, because of lower backscattered intensity, than for the brighter samples 0936 and 1309 (Bitenc et al., 2019).

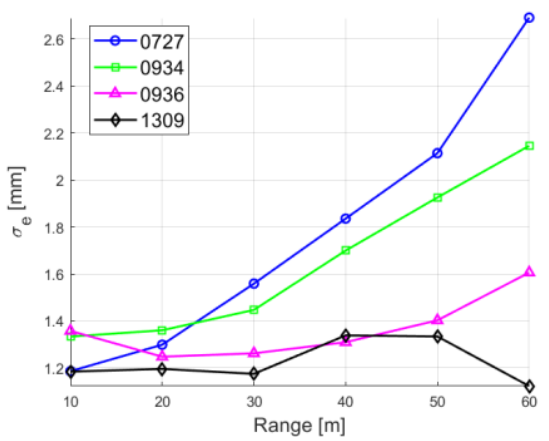

Fig. 4. Estimated noise, $\sigma_{e}$, for the four rock samples versus range

4.3.3 Noise elimination: The success of range denoising using SWT with global penalised high threshold is shown in Fig. 5. For all samples, a considerable amount of noise is removed, compared to the error $_{k}$ of noisy TLS surfaces shown in Fig. 3. The applied wavelet denoising method successfully removes the noise for samples 0727 and 0934; the error $_{k}$ is within 12 and $28 \%$. The Grasselli parameter of sample 0936 is overestimated up to $39 \%$ and underestimated for more than $31 \%$, for smaller $R(10 \mathrm{~m})$ and larger $R(50$ and $60 \mathrm{~m})$, respectively. For the roughest sample 1309, the Grasselli parameter is underestimated up to $57 \%$. Possible reasons for underestimated roughness of rougher samples 0936 and 1309 scanned from longer ranges are firstly, high thresholds remove also some surface details and secondly, the effective resolution, which decreases with the $R$, additionally smoothes surface details. High error $_{k}$ for sample 0936 scanned from $10 \mathrm{~m}$ could be a result of higher noise due to the strong reflection (Bitenc et al., 2019).

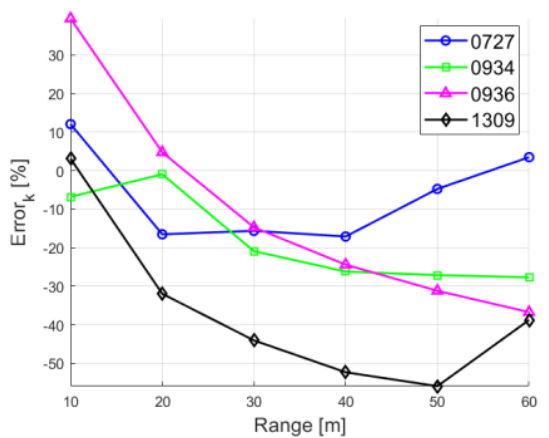

Fig. 5. Mean relative difference of Grasselli parameters $\left(\right.$ error $\left._{k}\right)$ for range denoised TLS surfaces using SWT with global penalised high threshold of the four samples versus range 


\begin{tabular}{ccc|cccc|cccc}
\hline$R$ & $\delta$ & $E I F O V$ & \multicolumn{4}{|c|}{$\lambda_{\min 1}[\mathrm{~mm}]$} & \multicolumn{4}{c}{$\lambda_{\min 2}[\mathrm{~mm}]$} \\
$\mathrm{m}$ & $\mathrm{mm}$ & $\mathrm{mm}$ & 0727 & 0934 & 0936 & 1309 & 0727 & 0934 & 0936 & 1309 \\
\hline 10 & 10 & 8.6 & 0.9 & 3.9 & $\mathrm{NaN}$ & 0.9 & 5.9 & 4.1 & 4.1 & 2.5 \\
20 & 13 & 11.2 & 2.7 & 1.6 & 1.1 & 4.7 & 5.9 & 4.1 & 4.9 & 4.1 \\
30 & 16 & 13.8 & 2.7 & 10.4 & 2.8 & 7.8 & 5.9 & 5.4 & 5.4 & 5.9 \\
40 & 19 & 16.4 & 2.9 & 14.3 & 5.1 & 12.6 & 7.8 & 9.8 & 8.2 & 7.6 \\
50 & 22 & 18.9 & 1.4 & 15.1 & 8.9 & 15.5 & 7.8 & 12.3 & 12.3 & 7.6 \\
60 & 25 & 21.5 & 1.1 & 17.4 & 13.1 & 6.7 & 15.7 & 12.3 & 9.8 & 10.6 \\
\hline
\end{tabular}

Table 3. Footprint size $\delta$, EIFOV and $\lambda_{\min }$ values for similarity measures $\operatorname{error}_{k, j}$ and $\operatorname{std}\left(\Delta Z_{k, j}\right)$ as a function of scanning range $R$

4.3.4 Effective resolution estimation: TLS effective resolution in regard to irregular surface detail extraction is studied by calculating similarity measures error $_{k, j}$ and $\operatorname{std}\left(\Delta Z_{k, j}\right)$ for denoised TLS surfaces acquired at scanning ranges $R_{k}$ and reference grid sizes $\lambda_{j}$. The similarity measures for the roughest sample 1309, which comprehends surface details on different scales, are shown in Fig. 6, as representative values for the other three samples.

error $_{k, j}$ is negative for smaller $\lambda_{j}$ and positive for larger; this is, the Grasselli parameter of TLS denoised surfaces is underestimated and overestimated compared to reference grids of smaller and larger $\lambda_{j}$, respectively. For each $R_{k}$, the grid size for error $_{k, j}=0$ is interpolated, which then represents the $\lambda_{\text {min }}$ (denoted by $\lambda_{\min l}$ ).

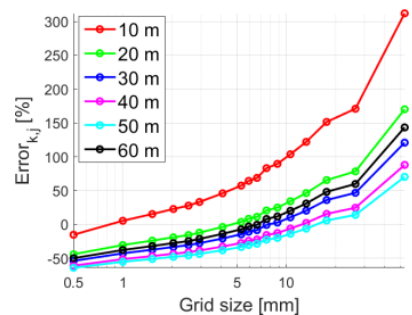

(a)

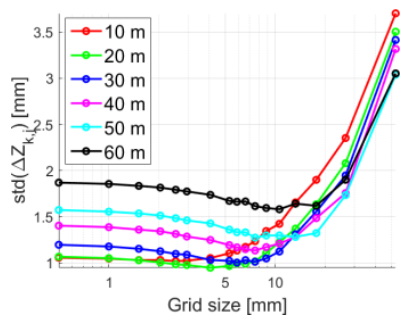

(b)
Fig. 6. Representative similarity measures for sample 1309 versus grid size, $\lambda_{j}$ (a) Mean relative difference of Grasselli parameters, error $_{k, j}$, and (b) standard deviation of height differences, $\operatorname{std}\left(\Delta Z_{k, j}\right)$

The $\operatorname{std}\left(\Delta Z_{k, j}\right)$ is relatively constant for smaller $\lambda_{j}$ and steeply increases for larger $\lambda_{j}$. The $\lambda_{j}$ when the $\operatorname{std}\left(\Delta Z_{k, j}\right)$ reaches its global minimum is taken as the $\lambda_{\min }$ at $R_{k}$ (denoted by $\lambda_{\min 2}$ ). Values $\lambda_{\min 1}$ and $\lambda_{\min 2}$ are summarised for all four samples in Table 3, where also the theoretical values of $\delta$ and EIFOV are given with respect to $R$.

$\lambda_{\min 1}$ shows the expected behavior for samples 0934, 0936 and 1309 ; it mostly increases with the $R$. The outliers appear for sample 0934 and 0936 at $10 \mathrm{~m}$, and sample 1309 at $60 \mathrm{~m}$, when the $\lambda_{\min 1}$ is too large, could not be defined since the error $_{1, j}$ does not reach zero and is too small, respectively. The outlier of sample 1309 can be explained with denoising results shown in Fig. 5. The surface is not thresholded high enough and stays rougher than expected resulting in smaller $\lambda_{\min 1}$

$\lambda_{\min 2}$ increases with $R$ for all samples, except for 0936 at $60 \mathrm{~m}$. It is the smallest for the roughest sample 1309 at almost all $R$, since this sample comprehends the smallest details.

The expectation that $\lambda_{\min }$ at certain $R$ is similar for all samples, if they comprehend details of similar scale, is partly met for $\lambda_{\min 2}$ values, whereas $\lambda_{\min 1}$ values when Grasselli parameters are compared vary a lot among samples. The reason is the sensitivity of Grasselli parameter to surface representation, thus to remnants of TLS noise and wavelet denoising artefacts (Bitenc et al., 2015a; Donoho and Johnstone, 1995).

To study a functional relationship between known theoretical and in this research developed empirical estimate of effective resolution, in Fig. 7, $\lambda_{\min 1}$ and $\lambda_{\min 2}$ are plotted versus EIFOV, and a linear regression curve $(y=p 1 \times x+p 2)$. The goodness of fit, the coefficient of determination $\rho^{2}$, and the parameters of linear model (slope $p 1$ and intercept $p 2$ ) are written in Table 4 .

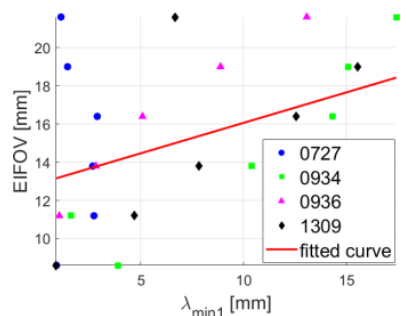

(a)

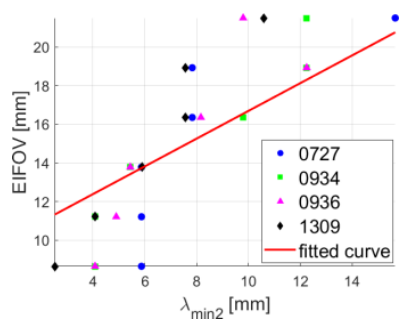

(b)
Fig. 7. Smallest extractable detail size $\lambda_{\min }$ versus EIFOV (a)

$$
\lambda_{\min 1} \text { and (b) } \lambda_{\min 2}
$$

$\lambda_{\min 2}$ shows stronger linear relation to EIFOV, this is having higher $\rho^{2}$ then $\lambda_{\min 1}$. Therefore $\lambda_{\min 2}$ can be considered as a more reliable measure of effective resolution on irregular surface. For the analysed $R$ from 10 to $60 \mathrm{~m}, \lambda_{\text {min }}$ is smaller than EIFOV. Shorter is the $R$, more pessimistic is the theoretical value EIFOV.

\begin{tabular}{ccc}
\hline & $\lambda_{\min 1}$ & $\lambda_{\min 2}$ \\
\hline$\rho^{2}$ & 0.9771 & 0.9813 \\
$\mathrm{p} 1$ & 0.3195 & 0.7183 \\
$\mathrm{p} 2$ & 12.87 & 9.509 \\
\hline
\end{tabular}

Table 4. Coefficients of determination, $\rho^{2}$, and parameters of fitted curves $(y=p 1 \times x+p 2)$ shown in Fig. 7

\section{CONCLUSIONS}

In this paper TLS potential and limitations for detailed and reliable rock discontinuity roughness estimation were investigated; this is the noise and resolution effect were analysed. The noise was successfully estimated and removed in the direction of range measurements applying Stationary Wavelet Transform (SWT) with penalised high threshold. An empirical method was developed to obtain the size of the smallest surface detail extractable from TLS data. This size was related to the theoretical value of effective resolution, the EIFOV.

By systematically comparing reference ATOS surfaces to original and denoised TLS surfaces, the influence of TLS data noise and resolution have been quantified, and the success of 
wavelet denoising has been demonstrated. The analyses have shown a high roughness over-estimation due to the TLS noise, especially for smoother surfaces. By applying wavelet denoising procedures, TLS data were substantially improved and more reliable estimates of rock surface roughness were obtained. However, the success highly depends on the threshold value with respect to surface roughness. Further study on optimal threshold value is needed.

The TLS range noise is not precisely known a priori and depends on surface reflectivity and scanning geometry. In this research the noise, $\sigma_{e}$, was estimated with the Median Absolute Deviation of the first level detail coefficients obtained from the SWT of range images. Additional testing of $\sigma_{e}$ in regard to a known range noise would be beneficial, in order to rely on $\sigma_{e}$ values.

For effective resolution analysis we assumed negligible smoothing effect of wavelet denoising and noise free TLS surface. However, the applied wavelet denoising method leaves some outliers (spikes) in surfaces, which should be removed with additional filtering step.

Comparative analysis of denoised TLS surfaces with the reference surfaces of known resolution quantified the smallest extractable detail on irregular rock surface, $\lambda_{\text {min }}$. Analyzing similarity of Grasselli parameters and height differences, the later measure resulted in more reliable estimates of $\lambda_{\text {min }}$. This empirically estimated effective resolution was related to theoretical value EIFOV and the EIFOV was found to be too pesimistic, especially for shorter ranges. In the futur, other similarity measures, as wavelet coherence, could be tested and the methodology should be approved on a larger set of TLS data, including longer ranges.

\section{ACKNOWLEDGEMENTS}

The authors would like to acknowledge the Slovenian National Building Institute and Civil Engineering Institute that enabled data acquisition with ATOS measuring system.

\section{REFERENCES}

Birgé, L., Massart, P., 1997. From Model Selection to Adaptive Estimation, in: Pollard, D., Torgersen, E., Yang, G.L. (Eds.), Festschrift for Lucien Le Cam. Springer New York, pp. 55-87.

Bitenc, M., Kieffer, D.S., Khoshelham, K., 2019. Range Versus Surface Denoising of Terrestrial Laser Scanning Data for Rock Discontinuity Roughness Estimation. Rock Mech. Rock Eng. doi.org/10.1007/s00603-019-01755-2

Bitenc, M., Kieffer, D.S., Khoshelham, K., 2016. Evaluation of Wavelet and Non-Local Mean Denoising of Terrestrial Laser Scanning Data for Small-Scale Joint Roughness Estimation. Int Arch Photogramm Remote Sens Spat. Inf. Sci XLI-B3, 181-186. doi.org/10.5194/isprs-archives-XLI-B3-181-2016

Bitenc, M., Kieffer, D.S., Khoshelham, K., 2015a. Evaluation of Wavelet Denoising Methods for Small-Scale Joint Roughness Estimation Using Terrestrial Laser Scanning. ISPRS Ann. Photogramm. Remote Sens. Spat. Inf. Sci. II-3/W5, 81-88. doi.org/10.5194/isprsannals-II-3-W5-81-2015

Bitenc, M., Kieffer, D.S., Khoshelham, K., 2015b. Estimating joint roughness using wavelet-based denoised terrestrial laser scanning data, in: Future Development of Rock Mechanics. Austrian Society for Geomechanics, pp. 517-522.

Bitenc, M., Kieffer, D.S., Khoshelham, K., Vezočnik, R., 2015c. Quantification of Rock Joint Roughness Using Terrestrial Laser Scanning, in: Lollino, G., Giordan, D., Thuro, K., Carranza-Torres, C., Wu, F., Marinos, P., Delgado, C. (Eds.), Engineering Geology for Society and Territory - Volume 6: Applied Geology for Major Engineering Projects. Springer International Publishing, Cham, pp. 835-838.

Buades, A., Coll, B., Morel, J., 2005. A Review of Image Denoising Algorithms, with a New One. Multiscale Model. Simul. 4, 490-530. doi.org/10.1137/040616024

Buades, A., Coll, B., Morel, J.M., 2004. On image denoising methods. Technical Note, CMLA (Centre de Mathematiques et de Leurs Applications.

Buckley, S.J., Howell, J.A., Enge, H.D., Kurz, T.H., 2008. Terrestrial laser scanning in geology: data acquisition, processing and accuracy considerations. J. Geol. Soc. 165, 625638. doi.org/10.1144/0016-76492007-100

CloudCompare, 2018. CloudCompare - Subsample tool. URL http://www.cloudcompare.org/doc/wiki/index.php?title=Edit $\% 5$ CSubsample (accessed 6.11.18).

Coifman, R.R., Donoho, D.L., 1995. Translation-invariant denoising. Springer, New York.

Donoho, D.L., 1995. De-noising by soft-thresholding. Inf. Theory IEEE Trans. Inf. Theory 41, 613-627. doi.org/10.1109/18.382009

Donoho, D.L., Johnstone, I.M., 1995. Adapting to unknown smoothness via wavelet shrinkage. J. Am. Stat. Assoc. 90, 12001224.

Fardin, N., Stephansson, O., Feng, Q., 2004. Application of a new in situ 3D laser scanner to study the scale effect on the rock joint surface roughness. Int. J. Rock Mech. Min. Sci. 41, 329335.

Gordon, S.J., Lichti, D.D., 2007. Modeling Terrestrial Laser Scanner Data for Precise Structural Deformation Measurement. J. Surv. Eng. 133, 72-80. doi.org/10.1061/(ASCE)07339453(2007)133:2(72)

Grasselli, G., 2001. Shear strength of rock joints based on quantified surface description (Ph.D. thesis). EPF Lausanne, Lausanne. doi.org/10.5075/epfl-thesis-2404

Grasselli, G., Wirth, J., Egger, P., 2002. Quantitative threedimensional description of a rough surface and parameter evolution with shearing. Int. J. Rock Mech. Min. Sci. 39, 789800. doi.org/10.1016/s1365-1609(02)00070-9

Gyaourova, A., Kamath, C., Fodor, I.K., 2002. Undecimated wavelet transforms for image de-noising. Rep. Lawrence Livermore Natl. Lab CA 18.

Hartzell, P.J., Gadomski, P.J., Glennie, C.L., Finnegan, D.C., Deems, J.S., 2015. Rigorous error propagation for terrestrial laser scanning with application to snow volume uncertainty. $J$. Glaciol. 61, 1147-1158. doi.org/10.3189/2015JoG15J031 
Khoshelham, K., Altundag, D., Ngan-Tillard, D., Menenti, M., 2011. Influence of range measurement noise on roughness characterization of rock surfaces using terrestrial laser scanning. Int. J. Rock Mech. Min. Sci. 48, 1215-1223. doi.org/10.1016/j.ijrmms.2011.09.007

Kregar, K., Grigillo, D., Kogoj, D., 2013. High precision target center determination from a point cloud. ISPRS Ann. Photogramm. Remote Sens. Spat. Inf. Sci. II-5/W2,139-144. doi.org/10.5194/isprsannals-II-5-W2-139-2013

Kulatilake, P., Balasingam, P., Park, J., Morgan, R., 2006. Natural rock joint roughness quantification through fractal techniques. Geotech. Geol. Eng. 24, 1181-1202. doi.org/10.1007/s10706-005-1219-6

Lichti, D.D., Gordon, S.J., 2004. Error Propagation in Directly Georeferenced Terrestrial Laser Scanner Point Clouds for Cultural Heritage Recording. Presented at the FIG Working Week 2004.

Lichti, D.D., Jamtsho, S., 2006. Angular resolution of terrestrial laser scanners. Photogramm. Rec. 21, 141-160. doi.org/10.1111/j.1477-9730.2006.00367.x

Mikhail, E.M., 1976. Observations and least squares. IEP-A Dun-Donnelley Publisher, New York.

Pesci, A., Teza, G., Bonali, E., 2011. Terrestrial Laser Scanner Resolution: Numerical Simulations and Experiments on Spatial Sampling Optimization. Remote Sens. 3, 167-184. doi.org/10.3390/rs3010167

Pollyea, R.M., Fairley, J.P., 2011. Estimating surface roughness of terrestrial laser scan data using orthogonal distance regression. Geology 39, 623-626. doi.org/10.1130/G32078.1

Poropat, G., 2009. Measurement of surface roughness of rock discontinuities, in: Proc the 3rd CANUS Rock Mechanics Symposium. Toronto.

Priest, S.D., 1993. Discontinuities and rock deformability, in: Discontinuity Analysis for Rock Engineering. Springer Netherlands, pp. 300-339.

Rahman, Z., Slob, S., Hack, R., 2006. Deriving roughness characteristics of rock mass discontinuities from terrestrial laser scan data. Presented at the Engineering geology for tomorrow's cities. 10th IAEG Congress.

Rengers, N., 1970. Influence of surface roughness on the friction properties of rock planes, in Proc. of the second Congress of International Society of Rock Mechanics, pp. 1-31.
Salmon, J., 2010. On Two Parameters for Denoising With NonLocal Means. IEEE Signal Process. Lett. 17, 269-272. doi.org/10.1109/LSP.2009.2038954

Schulz, T., Ingensand, H., Wunderlich, T., 2008. Calibration of a terrestrial laser scanner for engineering geodesy (Ph.D. thesis). Institut für Geodäsie und Photogrammetrie an der Eidgenössischen Technischen Hochschule Zürich. doi.org/10.3929/ethz-a-005368245

Smigiel, E., Alby, E., Grussenmeyer, P., 2011. TLS data denoising by range image processing. Photogramm. Rec. 26, 171-189. https://doi.org/10.1111/j.1477-9730.2011.00631.x

Soudarissanane, S., Lindenbergh, R., Menenti, M., Teunissen, P., 2011. Scanning geometry: Influencing factor on the quality of terrestrial laser scanning points. ISPRS J. Photogramm. Remote Sens. 66, 389-399. doi.org/10.1016/j.isprsjprs.2011. 01.005

Starck, J.-L., Elad, M., Donoho, D.L., 2004. Redundant Multiscale Transforms and Their Application for Morphological Component Separation, in: Advances in Imaging and Electron Physics. p. 400.

Tatone, B., Grasselli, G., 2012. Quantitative Measurements of Fracture Aperture and Directional Roughness from Rock Cores. Rock Mech. Rock Eng. 45, 619-629. doi.org/10.1007/s00603011-0219-5

Tesfamariam, E.K., 2007. Comparing discontinuity surface roughness derived from 3D terrestrial laser scan data with traditional field-based methods (MSc thesis). International Institute for Geo-information Science and Earth Observation, Enchede, The Netherlands.

Vezočnik, R., Bitenc, M., 2011. The use of terestrial laser scanning technology in tunneling, in: Likar, J. (Ed.). Presented at the The 10th International Symposium on Tunnel Construction and Underground Structures, Faculty of Natural Sciences and Engieneering, Ljubljana, Slovenia, 111-116.

Wujanz, D., Burger, M., Mettenleiter, M., Neitzel, F., 2017. An intensity-based stochastic model for terrestrial laser scanners. ISPRS J. Photogramm. Remote Sens. 125, 146-155. doi.org/10.1016/j.isprsjprs.2016.12.006

Zhang, Y., Liu, J., Li, M., Guo, Z., 2014. Joint image denoising using adaptive principal component analysis and self-similarity. Inf. Sci. 259, 128-141. doi.org/10.1016/j.ins.2013.08.002 